\title{
Laboreal
}

Volume $15 \mathrm{~N}^{\circ} 1$ | 2019

Trabalho e cooperação

\section{Gestión del riesgo y relaciones laborales en la industria de pasta de celulosa en el Uruguay}

Gestão de riscos e relações de trabalho na indústria de polpa de celulose no

Uruguai

Gestion des risques et relations de travail dans l'industrie de la pâte de cellulose en Uruguay

Risk management and labor relations in the cellulose pulp industry in Uruguay

\section{Francisco Pucci, Soledad Nión y Valentina Pereyra}

\section{(2) OpenEdition}

\section{Journals}

Edición electrónica

URL: http://journals.openedition.org/laboreal/1657

DOI: $10.4000 /$ laboreal. 1657

ISSN: 1646-5237

\section{Editor}

Universidade do Porto

\section{Referencia electrónica}

Francisco Pucci, Soledad Nión y Valentina Pereyra, « Gestión del riesgo y relaciones laborales en la industria de pasta de celulosa en el Uruquay », Laboreal [En línea], Volume $15 N^{0} 1$ | 2019, Publicado el 01 julio 2019, consultado el 10 abril 2020. URL : http://journals.openedition.org/laboreal/1657 ; DOI : https://doi.org/10.4000/laboreal.1657

Este documento fue generado automáticamente el 10 abril 2020. 


\section{Gestión del riesgo y relaciones laborales en la industria de pasta de celulosa en el Uruguay}

Gestão de riscos e relações de trabalho na indústria de polpa de celulose no

Uruguai

Gestion des risques et relations de travail dans l'industrie de la pâte de cellulose en Uruguay

Risk management and labor relations in the cellulose pulp industry in Uruguay

Francisco Pucci, Soledad Nión y Valentina Pereyra

\section{NOTA DEL EDITOR}

Manuscrito recibido en noviembre/2018

Aceptado tras peritaje abril/2019

\section{Introducción}

El trabajo se propone realizar una primera aproximación al estudio de dos plantas de producción de pasta de celulosa que han tenido alto impacto en la realidad industrial uruguaya. Ambas plantas, de origen internacional, pero con diferencias importantes en el funcionamiento de sus casas matrices, son consideradas, en términos de seguridad, como ejemplos de modelos de High Reliability Organizations (HRO) de la producción industrial nacional. En ambos casos las casas matrices imprimen modelos de gestión de riesgos industriales, cuyo eje pasa por el desarrollo de relaciones de confianza interna y externa, aunque con adaptaciones al medio en el que cada empresa opera. Estas adaptaciones, a su vez, van a tener soluciones específicas en función de las diferencias en los modelos originales de cada empresa. La hipótesis que se sostiene en este trabajo 
es que estos modelos requieren para su implementación un modelo de relaciones laborales diferente al que predomina en la mayoría de las empresas nacionales. Las altas condiciones de seguridad que se desarrollan en el sector están basadas en una estrategia de diálogo permanente por parte de la gerencia con un sindicato con una fuerte orientación corporativa, que prioriza las metas económicas y que tiende a reducir el componente ideológico en sus formas de acción. Estas orientaciones marcan importantes diferencias con las formas de acción predominantes en el sindicalismo uruguayo.

\section{Metodología}

2 El trabajo forma parte de la investigación "Organización del trabajo y gestión del riesgo en la industria de producción de pasta de Celulosa" aún en curso, financiada por la Comisión Sectorial de Investigación Científica de la Universidad de la República Oriental del Uruguay. La misma está basada en una estrategia cualitativa donde se realizó un mapeo de actores vinculados al sector, estrategias conversacionales de recogida de datos con informantes calificados y casos muestreados teóricamente, análisis de fuentes secundarias sobre el complejo celulósico forestal, intercambios en paneles de expertos y observación no participante.

3 El mapeo de actores tuvo en cuenta la incorporación de representantes de diversas visiones en torno a los riesgos presentes en la producción de pasta de celulosa en nuestro país. Las reflexiones presentadas en este trabajo se realizan a partir de un primer análisis de 18 entrevistas (individuales o colectivas) a diferentes informantes calificados realizadas en el correr del año 2017 y comienzos de 2018. En estas instancias participaron dirigentes sindicales sectoriales, Técnicos Prevencionistas, jerarcas de organismos estatales vinculados a la temática, expertos en derecho ambiental, referentes académicos con estudios en la temática medio ambiental y de salud humana, encargados de seguridad de las empresas que se analizarán y personas vinculadas a Organizaciones Sociales Civiles ambientalistas.

4 Para el trabajo de campo, a partir de un muestreo teórico abierto y flexible, se seleccionaron diferentes actores sociales que se corresponden con grupos sociales relacionados más o menos directamente a la producción de pasta de celulosa en dimensiones tales como la productiva, laboral, social, ambiental y de salud. Como tales, representan visiones técnicas, políticas, comunitarias, con diferente injerencia en la definición de los modelos de producción, la gestión del riesgo y de las condiciones laborales, impacto tecnológico- productivo, y salud y seguridad en el sector.

5 Este trabajo se propone exponer los resultados de estas entrevistas, como primer análisis preliminar de los procesos de gestión del riesgo que se desarrollan en estas organizaciones, su adaptación al entorno local, las características de sus procesos de trabajo, los mecanismos formales e informales de regulación y su impacto en las formas de acción sindical. Estas empresas desarrollan sus actividades con niveles tecnológicos muy superiores a las demás industrias de la rama, pagan salarios más altos que el promedio de los trabajadores uruguayos, y despliegan sus actividades en un marco institucional abierto al diálogo y a la negociación. Estas características impactan no sólo en las condiciones de trabajo y de seguridad sino también en las formas de acción colectiva de reivindicación y negociación laboral, que adquieren funcionamientos muy diferentes a las formas tradicionales de acción sindical del Uruguay. 


\section{Las organizaciones de alta confiabilidad}

En su teoría de los accidentes normales, Perrow (1984) desarrolló la tesis de que los accidentes graves son la consecuencia inevitable de sistemas cuyas características intrínsecas son la complejidad de las interacciones y las fuertes interdependencias entre las partes del sistema. La combinación de estos dos factores produce una mezcla explosiva que conduce inevitablemente a accidentes y catástrofes. Esta perspectiva pesimista fue discutida por la teoría de las High Reliability Organizations (HRO) que sostiene básicamente la idea de que las organizaciones complejas y de alto riesgo pueden reducir sensiblemente las posibilidades de accidentes importantes para los trabajadores y para el entorno.

Desde esta perspectiva, la clave para lograr altos niveles de fiabilidad es la capacidad de construir confianza social e institucional entre sus miembros, en los organismos públicos y privados externos y en el público en general. La Porte (2001) muestra que no todas las organizaciones tienen exigencias de alta fiabilidad. Estas exigencias, en general están presentes en organizaciones cuyos errores o accidentes pueden tener las siguientes consecuencias:

- Una duda importante sobre su viabilidad económica, si la calidad de la cadena de producción es cuestionada;

- Una disminución significativa de su capacidad para responder a misiones de interés público o nacional, como la defensa de la seguridad de las poblaciones;

- Daños importantes para los operadores, si son afectados físicamente de manera inmediata o latente;

- Daños importantes para las personas que habitan en las proximidades de la organización;

- Daños importantes al medio ambiente que afectan las condiciones de vida de los habitantes y de manera más durable, al ecosistema.

Las posibilidades de construir altos niveles de confianza interna están fuertemente asociados, desde esta perspectiva, al funcionamiento y características centrales de organizaciones. Fahlbruch y Wilpert (1999) definen los rasgos centrales asociados a las organizaciones de tipo HRO:

- Un sentido agudo de su misión y de su reconocimiento

- Un nivel elevado de competencia y de performance

- Una estructura flexible que da cuenta de las redundancias del sistema

- Un modo colectivo de ejercicio del poder que permite flexibilizar el proceso de toma de decisiones

- Una búsqueda continua del progreso sobre la base de la experiencia

- Sistemas destinados a recompensar la detección de errores y la circulación de informaciones al respecto

- Una cultura organizacional de fiabilidad

El logro de altos niveles de fiabilidad externa depende fundamentalmente de las relaciones que estas organizaciones mantienen con los organismos públicos y con las instituciones y organizaciones de su entorno. Estas son las que son las que les confieren una legitimidad pública y un componente de confianza en amplios públicos. Para construir espacios de confianza institucional externa, las organizaciones de alta fiabilidad deben tener una participación constante en los espacios de deliberación y consulta con los actores externos, sociales e institucionales, y deben aplicar 
efectivamente los acuerdos que se alcanzan con los mismos. La condición más importante para establecer relaciones de confianza tanto interna como externa lo constituye la construcción de una credibilidad en el largo plazo, lo que implica que la constancia en los esfuerzos por alcanzar la fiabilidad son motores centrales para estos fines (La Porte, 2001).

Por otra parte, como indica Rochlin (1996) una organización que funciona de manera fiable, segura y eficiente, no se diferencia de otras organizaciones por un conjunto particular de reglas o procedimientos, o por competencias específicas en formación y gestión. La seguridad es ante todo, para este autor, una propiedad que emerge de las relaciones, ritos y mitos que conforman al conjunto de la organización. En esta línea, Douglas (1985) mostró que el riesgo no es una propiedad inmodificable del entorno, sino que está socialmente definida y varía en función de las relaciones sociales y de los comportamientos colectivos. Beck (1998) y Luhmann (1992) también mostraron que la percepción y evaluación de los riesgos está condicionado socialmente y que forman parte de las luchas sociales en las sociedades modernas.

11 Desde otra perspectiva, se considera que la fiabilidad no es únicamente una propiedad de las organizaciones, ni el producto de las interrelaciones entre los actores (Bourrier, 2001). La misma resulta más bien de un acoplamiento exitoso entre la organización y sus miembros y entre la organización y su entorno. La redundancia de los canales de decisión, la redundancia del control entre los actores, las actividades permanentes de reciclaje y de entrenamiento, la adhesión de los miembros a los fines de la organización y la delegación de poder conviviendo con poderes centralizados son los principales criterios que permiten distinguir a las organizaciones altamente fiables.

Boissières (2007), a su vez, pone de relieve la necesidad de contar con diferentes enfoques sobre la fiabilidad organizacional. A primera vista, nos encontramos con dos modos aparentemente irreconciliables de regulación del riesgo. Por un lado, encontramos una lógica securitaria, que tiene una perspectiva lineal de los accidentes, para la cual la única solución posible es la adición de medidas de seguridad parciales que redundarían en un aumento de la seguridad total del sistema. Esta perspectiva la podemos encontrar en las teorías del error humano de Reason, que construyen sistemas defensivos para evitar que la organización se perjudique por los desvíos humanos y organizacionales. La base de esta teoría es una profunda desconfianza hacia los operadores y hacia la organización, que obliga a generar barreras de protección contra los mismos.

13 La alternativa a esta perspectiva es la teoría de gestión de los riesgos, que asume que el riesgo es una probabilidad siempre presente en las organizaciones, y que las decisiones sobre el mismo no son exclusivamente técnicas. En la misma entran en juego la lógica de los actores, sus representaciones y sus interesses (Dourlens et al., 1991). Esta perspectiva intenta superar una visión mecánica y lineal del riesgo, asumiendo el carácter complejo de su gestión y la necesidad de desarrollar competencias individuales y colectivas para reducir sus posibilidades.

14 Desde la perspectiva securitaria, la seguridad reposa en las jerarquías, mientras que desde la lógica de la gestión del riesgo este es el principal factor de inseguridad. A la inversa, para la primera perspectiva, las prácticas desviadas de los actores generan las perturbaciones más riesgosas para el sistema socio-técnico, en tanto que para la segunda perspectiva, estos desbordes son los que aseguran el funcionamiento seguro de 
las organizaciones. Esta oposición pone de manifiesto las dos grandes fuentes de regulación, la que surge de los actores y la que proviene del sistema.

Boissières desarrolla el concepto de robustez organizacional, como alternativa para superar la oposición de estos dos modos de regulación del riesgo. Esta perspectiva privilegia el análisis de los riesgos a partir del estudio de las perturbaciones de la organización. La necesidad de partir de las perturbaciones y no de las vulnerabilidades, como plantean las teorías precedentes, proviene de la existencia de una distancia entre la situación potencial, descripta por la evocación a las vulnerabilidades organizacionales, y la situación que se observa en concreto. Tomando en cuenta las perturbaciones como punto de partida, el análisis pasa de la eventualidad de grandes accidentes o catástrofes a los comportamientos cotidianos y a las posibilidades de actuar sobre los mismos. Aceptando las perturbaciones como realidades normales y omnipresentes en las actividades productivas, el desafío consiste en evitar que la perturbación termine en accidente. La reflexión sobre la robustez organizacional se refiere a las condiciones que tiene la organización para mantener un estado de seguridad aceptable y sobre la tolerancia del sistema a diferentes tipos de perturbaciones en el campo de la seguridad del sistema.

Las organizaciones de alta fiabilidad priorizan los procesos de construcción de confianza como pilares centrales de sus políticas de reducción de accidentes. Como señalan (Coriat \& Guennif, 2000), es al amparo de ciertas características de las organizaciones en las cuales actúan, que los actores son incitados o no a entrar en relaciones de confianza. Establecer relaciones de confianza requieren de procesos complejos y en esencia revocables que solo se pueden afirmar en la reciprocidad. En este sentido, las organizaciones deben generar las condiciones para que el proceso de construcción de confianza sea posible. La confianza, más que la autoridad, la jerarquía y la disciplina, permite reducir los niveles de incertidumbre de los sistemas y establecer formas de control más informales y laxas. Esta flexibilidad es la que genera las mejores condiciones para una eficaz gestión de la incertidumbre intrínseca de los riesgos.

17 A partir de estas premisas conceptuales que sintetizan la discusión sobre los modelos HRO de gestión del riesgo, analizaremos dos casos concretos de empresas que implementan sus políticas de seguridad sobre sus fundamentos. Previamente, realizaremos una contextualización del complejo económico en el cual se insertan estas empresas, para enmarcar el entorno económico y social en el cual se desarrollan, y luego avanzar en los procesos de gestión del riesgo de las mismas.

\section{Caracterización del complejo forestal - celulósico uruguayo}

El estudio está centrado en el análisis de dos empresas de producción de celulosa, lo que requiere una breve síntesis del desarrollo del sector en el Uruguay. La presencia más evidente del ingreso de transnacionales en el agro se dio de la mano de la expansión de las empresas forestales que, a fines de los años 90 se instalaron en nuestro país (Florit \& Piedracueva, 2016). Es en este contexto que deben entenderse las inversiones que tanto empresas de capital nacional como empresas multinacionales han realizado en el sector forestal y también en las etapas de transformación y procesamiento agroindustrial de la madera y la celulosa. 

las grandes empresas a delinear una suerte de «estrategia forestal internacional» por la cual deslocalizaron parte de su actividad productiva. Este proceso de deslocalización de la producción forestal reconoce dos etapas: en la primera, las grandes empresas expanden sus plantaciones en distintos lugares del mundo, de manera de proveer a sus plantas industriales ubicadas en el Norte con la celulosa producida en el Sur; en la segunda, cuando las plantaciones propias han alcanzado un volumen considerable $\mathrm{y}$, si la rentabilidad es favorable, comienzan a construir centros industriales en los nuevos países productores, lo que lleva, en algunos casos, al cierre de las plantas en los países de origen" (p. 78).

Juncal y Fernandez (2013), a su vez, proponen dividir la evolución del complejo forestal uruguayo en tres etapas: "una primera fase de instalación, que abarcaría el período comprendido entre la promulgación de la ley 15.939 (año 1987) donde la superficie de las plantaciones forestales era de 46.000 hectáreas y el año 2007 en que comienza a operar la primera planta de procesamiento de celulosa en el Uruguay y la superficie plantada pasa a ser de 850.000 hectáreas. La segunda fase está signada por una primera planta de celulosa funcionando con una capacidad instalada de 1,1 millón de toneladas de celulosa blanqueada de fibra corta de eucalipto y con un consumo de 3,6 millones de metros cúbicos al año". En esta segunda etapa también se instala la segunda planta productora de celulosa próxima a la localidad de Conchillas, en el departamento de Colonia (sobre el Río de La Plata). Por último, una tercera etapa -actual- que podría caracterizarse como de "expansión y la proyección de una tercera planta de procesamiento de pulpa de celulosa; en esta fase, además de la ampliación de la capacidad operativa de la fase industrial (plantas de celulosa), se requiere una red logística acorde a las demandas de la producción, tanto en lo que refiere a la posibilidad de acceso a distintos territorios, como a la articulación de distintas modalidades de transporte para viabilizar la producción forestal en zonas más alejadas de las plantas de procesamiento, "conquistando" nuevos territorios" (Juncal \& Fernandez, 2013, p. 44). del proceso de transnacionalización de las plantaciones y la implantación de empresas multinacionales permitieron el desarrollo de nuevas infraestructuras, reglamentaciones y pautas productivas y laborales en nuestro país, marcando la entrada a una forma diferente de producción (Florit, 2013).

El sector incluye al menos cuatro cadenas industriales de base forestal:

(1) la cadena celulósico-papelera que es la de mayor peso dentro del sector y en la cual participan empresas reconocidas mundialmente;

(2) la transformación mecánica: productos de madera elaborada, esta cadena se identifica por la coexistencia de empresas extranjeras y nacionales;

(3) la industria química;

(4) y la energética.

La fase secundaria o industrial, en la que centramos nuestro trabajo actual de investigación, comprende las actividades de trasformación de la madera realizada en diversas cadenas, incluida la comercialización; dentro de las actividades relacionadas con la fase industrial también se encuentra la generación de energía a través de subproductos forestales (biomasa forestal y subproductos de la transformación mecánica y química), que ha cobrado relevancia y posee una importante perspectiva de 
crecimiento debido al aumento de la oferta de materia prima y a políticas de Estado en el sector (Informe Uruguay XXI, 2017).

La evolución y la importancia de la actividad forestal-celulósica en el país se puede observar a través de diversos indicadores. Por ejemplo, Tommasino et al. (2016) observan que desde 1987 la superficie forestada se multiplicó por más de 6 veces al pasar de 186 mil en 1990 a 1,15 millones de hectáreas en 2016.En cuanto a la propiedad de las plantaciones, al año 2015 un $41 \%$ de la propiedad de plantaciones correspondía a fábricas de celulosa (Pou y Asociados, 2016). Asimismo, en ese año el PIB forestal representó $2,5 \%$ del PIB del país donde un $2,1 \%$ correspondió a la fase industrial y un $0,4 \%$ a la fase primaria (Tommasino et al., 2016).

Dentro de esta evolución es necesario destacar que la producción de celulosa se trasformó en la principal actividad industrial de la cadena; a modo de ejemplo, en el año 2010, la relación de madera cosechada destinada a la producción de celulosa, respecto a cualquier otro destino, era de 6 a 1 (Agenda Forestal, 2011).La magnitud del aporte de la actividad se evidencia cuando vemos que en el año 2015 la celulosa tuvo una participación del 64\% en el PIB de la fase industrial y del 1,3\% en el PIB nacional (Tommasino et al., 2016).Por otro lado, el desarrollo del sector en el país tuvo que ver también con los beneficios fiscales y el marco regulatorio de la actividad forestalcelulósico como factores decisivos para el desarrollo del complejo en nuestro país.

A nivel de I+D y tecnología, en la fase industrial, nuestro país ha innovado en materia de producción de pulpa de celulosa con tecnología que apunta a disminuir el impacto sobre el ambiente, y también en términos de aserraderos nacionales, con productos innovadores (Morales, 2016). El informe de Comité de Desarrollo y Propiedad Intelectual (CDPI) sobre las tecnologías en la primera transformación industrial señala dos principales procesos de invocación: "el proceso para incrementar la eficiencia y productividad de la actividad de los aserraderos, tableros y producción de celulosa e innovaciones para incrementar la oferta de energía renovable. Se trata de tecnologías relativamente estandarizadas mundialmente, las cuales son adquiribles vía mercado a un conjunto reducido de proveedores internacionales. Estas tecnologías suelen ser protegidas vía derechos de patentes, y son producidas por empresas que operan con escala mundial. La protección por patentes de esas tecnologías en un país determinado depende de la capacidad industrial del mismo de reproducirlas. Los esfuerzos locales se concentran, en consecuencia, en la introducción de innovaciones incrementales para adaptar la tecnología importada a las necesidades e insumos locales, y en el diseño de las plantas fabriles." (Anllo et al., 2013). Igualmente, otros factores que se introdujeron son el proceso de extensión y profundización de la certificación en la rama y las políticas de Responsabilidad Social Empresarial (RSE).

\subsection{Empresas "pasteras" y mano de obra}

31 Al momento, existen dos empresas encargadas de la producción de pasta de celulosa en el Uruguay. La primera, de origen finlandés, es una de las mayores empresas productoras de celulosa del mundo. En Uruguay, según datos relevados, emplea a 550 personas en forma directa y más de 2.800 en toda su cadena productiva (incluyendo viveros, plantaciones forestales, transporte, planta y puerto). Su complejo industrial está ubicado en Fray Bentos, departamento de Río Negro, y cuenta con capacidad de producción de 1,3 millones de toneladas de celulosa de fibra corta de eucalipto, 
destinada casi en tu totalidad a la exportación. Además, posee una unidad generadora de energía a partir de biomasa (Informe Uruguay XXI, 2016).

La segunda empresa se instala en el año 2009 y está conformada por partes iguales de dos empresas del sector forestal: una de capitales chilenos y otra sueco-finlandesa. Cuenta, entre campos propios y de terceros, con unas 145.000 hectáreas forestadas en 11 departamentos del país. El 35\% de los campos propios son áreas de conservación biológica, montes nativos o especies autóctonas en las que la empresa implementa planes de conservación y monitoreo. La planta está instalada en Conchillas (departamento de Colonia), a orillas del río de la Plata (Informe Uruguay XXI, 2017).

Paolino et al. (2014) señalan que si bien las plantas de celulosa articulan a nivel local la cadena de valor "aguas arriba" de la celulosa y están integradas en cadenas globales de valor (CGV), emplean a poco personal, aunque de alta calificación. No obstante, en las etapas de construcción de las plantas se da un alza significativa de la cantidad de empleos (Tomassino et al., 2016).

En Uruguay la población empleada en la industria de la pasta y el papel trabaja fundamentalmente a tiempo completo en estructuras de gestión tradicionales. A causa del alto costo de la inversión, muchas operaciones de la fabricación de pasta funcionan sin interrupción y requieren el trabajo por turnos. En términos generales la mayoría de los trabajadores cualificados y muchos de los no cualificados han recibido formación especializada para el desempeño dentro de la planta. Directivos y técnicos suelen tener titulación universitaria

En las fábricas más modernas, el trabajo se realiza desde salas de control independientes para aislar los equipos electrónicos del entorno ambiental de la producción de pasta y de papel donde se desempeñan los trabajadores. Estas salas cuentan con aire acondicionado, ofrecen refugio contra el ruido, las vibraciones, la temperatura, la humedad y la exposición a los agentes químicos inherentes a las operaciones fabriles, mejorando las condiciones tradicionales del sector forestal y de la industria maderera- papelera tradicional.

La evolución global del sector muestra una tendencia a la reducción de la accidentalidad en los últimos años (Ver Cuadro 1) Esta constatación surge del análisis de la información que es proporcionada por el Banco de Seguros del Estado, en la cual la producción de celulosa no se desagrega de la producción de madera y de papel. 
Cuadro 1 - Evolución de accidentes laborales 2014-2017 por rama de actividad
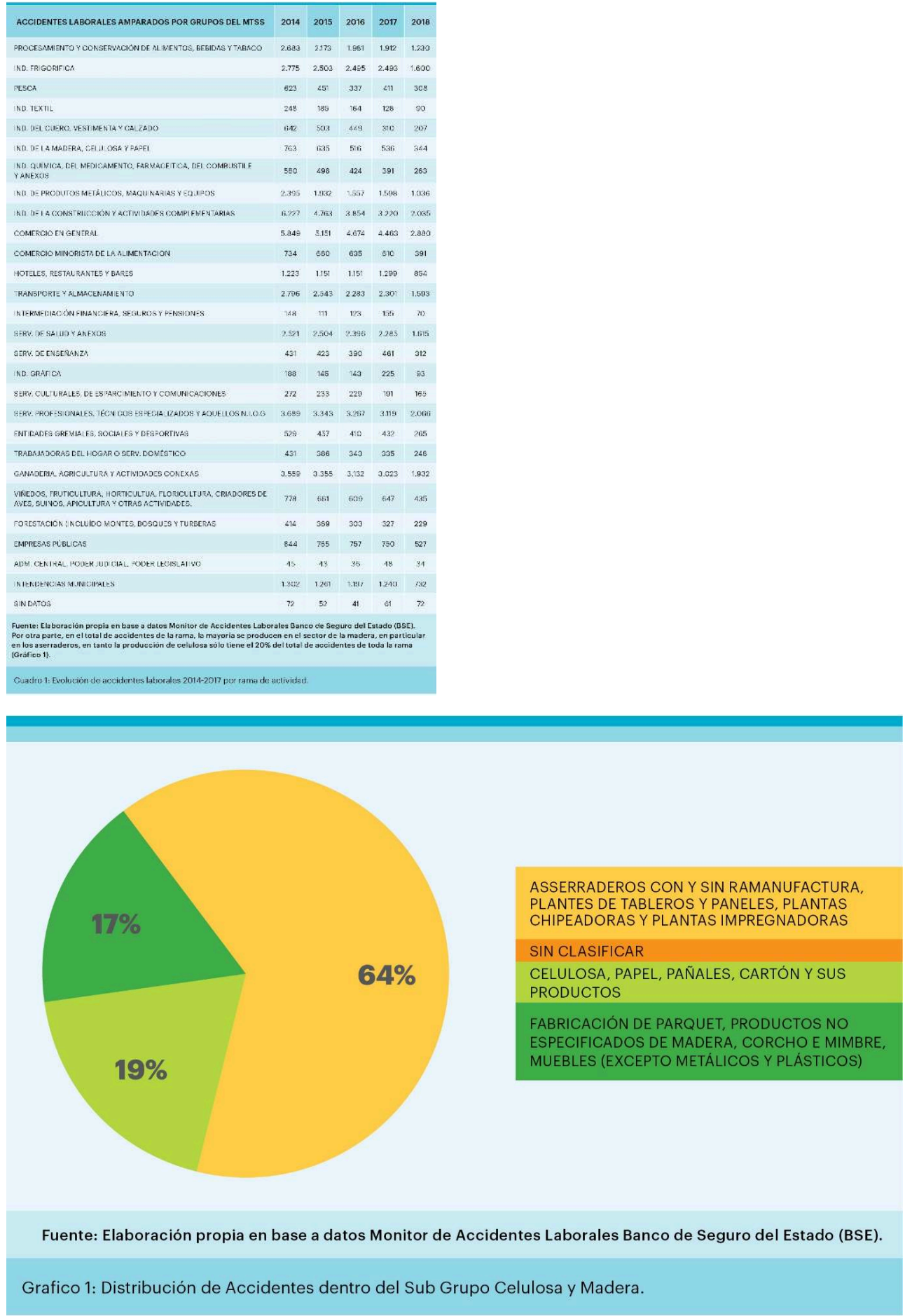

Por otra parte, en el total de accidentes de la rama, la mayoría se producen en el sector de la madera, en particular en los aserraderos, en tanto la producción de celulosa sólo tiene el $20 \%$ del total de accidentes de toda la rama (Gráfico 1).

En las entrevistas realizadas a los jerarcas de las empresas analizadas, los mismos alegaron que la frecuencia de accidentes en ambas empresas es muy baja. Los accidentes se producen fundamentalmente en las paradas de mantenimiento, en la cual se manipulan directamente los productos, y afectan principalmente a los trabajadores 
de las empresas tercerizadas. Estos datos no pueden ser corroborados estadísticamente, ya que no es posible acceder a los datos internos de las empresas. La única fuente son los datos estadísticos expuestos, que son agregados por rama y sector.

\section{La gestión del riesgo laboral} implementados por las dos empresas consideradas en este trabajo se inspiran en los modelos de seguridad de tipo HRO desarrollados más arriba, pero que han sufrido diferentes adaptaciones a las culturas locales de gestión y trabajo. El análisis comparado con otras experiencias de empresas HRO en América Latina se enfrenta a las dificultades de la escasez de estudios en la materia. De la Garza Toledo (2010) ha mostrado como las nuevas formas de organización del trabajo, inspiradas en el modelo "toyotista" de las casas centrales, tuvieron que adaptarse a las condiciones locales de cada país. La "nacionalización" de las prescripciones de las casas matrices también ocurre en los modelos de seguridad, que si bien mantienen las directrices centrales, sufren adaptaciones específicas a las realidades locales. Esto también ocurre con los modelos de seguridad. Cantero, y Seijo (2012) plantean la especificidad de las formas de HRO en América Latina, y desarrollan una tipología para dar cuenta de estas diferencias.

En el caso uruguayo, una primera adaptación se produce con la fusión de formas de gestión y culturas laborales que combinan componentes latinoamericanos y del norte de Europa, muy diferentes entre sí. El intercambio cultural con los países del norte de Europa se tradujo en una fuerte internalización en los trabajadores locales de la cultura laboral, la lógica de trabajo y el estricto cumplimiento de plazos propios del mundo laboral nórdico, bastante ajenos al perfil clásico de los trabajadores uruguayos. Más allá de estas influencias, las formas de gerenciamiento y de implementación de las políticas de seguridad tienen niveles importantes de autonomía, en el marco de la difusión de las herramientas de gestión a través de sus casas matrices.

Un segundo tipo de adaptación refiere al menor nivel de rigidez en la aplicación de las regulaciones de seguridad. En muchas ocasiones, los propios encargados parecen incumplir algunas normativas de seguridad para resolver problemas técnicos que se manifiestan en el proceso de trabajo, debido a la presión por alcanzar las metas productivas establecidas. Estos deslices implican ajustes cotidianos entre las reglas de seguridad y las condiciones específicas de trabajo toleradas y admitidas por las propias jerarquías de las empresas. De igual modo, persisten en los trabajadores locales "viejas mañas" propias de contextos productivos menos exigentes en seguridad: resistencia a los controles, al llenado de formularios y a las prescripciones a cumplir en el desempeño de cualquier tarea.

41 Un tercer ejemplo de adaptación refiere a las empresas subcontratadas, que si bien parecen haber mejorado sus niveles de seguridad por requerimiento de las empresas contratantes, mantienen niveles de inversión en seguridad inferiores, similar al promedio de las empresas nacionales.

42 Las entrevistas analizadas muestran también cuales son los mecanismos de construcción de confianza puestos en marcha por estas empresas. Como mostramos más arriba, uno de los ejes centrales de los modelos HRO es el desarrollo de la comunicación a todos los niveles de la empresa, lo que permite detectar errores o 
riesgos potenciales a través de la participación activa de los trabajadores. En las empresas pasteras analizadas se estimula que los trabajadores comuniquen los problemas de seguridad que perciben en sus tareas cotidianas y propongan alterativas de funcionamiento más convenientes. Los trabajadores cuentan con diversas vías de comunicación para plantear estos problemas de seguridad o de condiciones de trabajo (delegados de seguridad, jefes de área, departamento técnico). Por otra parte, existe una actitud receptiva por parte de los jerarcas a las mismas, lo que deriva, en ocasiones, en acciones para cambiar o mejorar la situación planteada.

Otro de los mecanismos asociados a los modelos HRO de gestión del riesgo es el desarrollo de un sistema de estímulos y recompensas que valoriza la trasmisión y puesta en común de las informaciones en relación a los posibles riesgos o errores en el desempeño cotidiano de las tareas. Este sistema, observado en las empresas pasteras analizadas, se concreta a través de bonificaciones especiales que se obtienen a cambio del logro de las metas definidas en seguridad y productividad. A través de la negociación colectiva con los sindicatos de trabajadores, las dos empresas analizadas acuerdan premios colectivos por productividad, calidad y accidentalidad. Uno de los objetivos de estos premios es llegar a niveles de accidentalidad cero, generando una conciencia general sobre el tema y la participación de todos los trabajadores para alcanzar buenos resultados en términos de seguridad. En este plano, las políticas de seguridad de las empresas analizadas superan las condiciones de buena parte de la producción forestal del Uruguay, en las cuales se premiaba únicamente las metas productivas, y de manera individual, oponiendo producción y seguridad.

Las empresas analizadas también introducen mecanismos de evaluación de las carreras laborales y de las oportunidades de ascenso personal que incluyen la temática de la seguridad. La evaluación de los desempeños personales se realiza en tres grandes dimensiones: desempeño cotidiano, conocimiento técnico e iniciativa. La formulación de propuestas o alternativas de seguridad forma parte de la evaluación del nivel de iniciativa individual, generando mejores puntajes y posibilidades de ascenso en la carrera laboral.

Otra dimensión que surge de las entrevistas a expertos se refiere a la formación del personal. Estas empresas desarrollan políticas de capacitación específicas en materia de seguridad y de aprendizaje permanente en el lugar de trabajo. Las empresas parecen apostar a una formación continua de tipo práctico a través de la puesta en común de los problemas a los que se enfrentan los trabajadores en el proceso productivo y a las necesidades y sugerencias que los propios trabajadores transmiten a las jerarquías laborales.

La última dimensión a tener en cuenta se refiere a las condiciones laborales. El proceso de trabajo de estas empresas establece condiciones laborales que favorecen la implementación de un modelo HRO de gestión de la seguridad, que se expresan en la intervención de pocos trabajadores en la producción de la pasta de celulosa, debido al automatismo del mismo. La mayor parte de los trabajadores desempeñan sus actividades en salas de control con máquinas automatizadas, desde las cuales analizan y modifican los parámetros requeridos para la producción. Estas condiciones de trabajo parecen reducir las probabilidades de accidentes típicos en otras empresas de la misma rama que no alcanzaban este nivel tecnológico. Se sustituyen riesgos traumatológicos y de quemaduras por riesgos ergonómicos y de mayor carga cognitiva. 
47 La intervención directa en el proceso de trabajo se da en las paradas de mantenimiento, que se realizan de manera rutinaria o cuando existen problemas técnicos puntuales. Para estas tareas, ingresa una cuantiosa plantilla de trabajadores externos. La presión para reducir los costos de las paradas de mantenimiento tiene como consecuencia la aceleración de los ritmos de realización de las tareas, lo que puede llevar a que el trabajador no tome las debidas precauciones en su trabajo, o que no cumpla de manera adecuada con las normas vinculadas a la ejecución de las tareas.

\section{Relaciones laborales y rol del sindicato}

El desarrollo de relaciones de confianza y de comunicación inherentes al modelo HRO de gestión del riesgo, requiere un sistema de relaciones laborales que priorice el diálogo y la negociación con las organizaciones sindicales que representan a los trabajadores de estas empresas. Esta orientación al diálogo y a la negociación por parte de la empresa exige como contrapartida una actitud similar por parte de las organizaciones sindicales, de manera de favorecer el desarrollo de un sistema de relaciones laborales con amplios espacios de consenso.

Los sindicatos de los trabajadores dedicados a la producción de celulosa forman parte de la rama que nuclea la producción de papel y de celulosa. Estas dos actividades están experimentando en la actualidad ciclos económicos opuestos: mientras la industria del papel está en extinción, el sector primario de producción de pasta de celulosa está en expansión. Esta dicotomía se traduce en diferencias salariales importantes entre los trabajadores de las empresas pasteras y sus semejantes que producen papel. Esta realidad económica, signada por altos sueldos pagados por empresas multinacionales, con altos niveles de inversión en todos los planos de la gestión de recursos humanos, fue el contexto de desarrollo del sindicalismo en el sector de pasta de celulosa. La especificidad y excepcionalidad de las condiciones de trabajo impactaron en la formación de los sindicatos de estas empresas, en sus formas de organización, en sus orientaciones ideológicas, programáticas y en sus acciones.

50 En la primera empresa analizada, el sindicato se formó en un contexto muy peculiar de cooperación con el sindicato de la casa matriz y con altos niveles de aceptación por parte de las jerarquías y gerencias de la empresa. El perfil de los trabajadores y sindicalistas de estas empresas es también diferente al promedio de la rama. Se trata de jóvenes con niveles de educación medio-altos y con promedios salariales muy superiores a la media de la rama de actividad. Para muchos de estos jóvenes, las condiciones excepcionales de trabajo en las que desempeñan sus tareas, despegadas de las que se encuentran en el resto del sector, y de los trabajadores industriales uruguayos en general, son la única realidad laboral que conocieron después de terminar sus estudios. En este contexto, el sindicato parece ser percibido como una especie de "club social" cuyo cometido es otorgar beneficios a los trabajadores, sin contenido ideológico o programático más amplio.

51 Estas condiciones también son diferentes a la que tienen los trabajadores de las empresas tercerizadas que trabajan para la pastera, que mantienen perfiles salariales similares al conjunto de la rama. El sindicato se ha preocupado especialmente por la situación de estos trabajadores, tratando de acercar a los mismos a la organización sindical y luchando por la ampliación de los beneficios que perciben los trabajadores de planta a este núcleo de trabajadores tercerizados. Un ejemplo en este sentido son los 
bonos de producción que la empresa paga por los resultados obtenidos a nivel productivo, ambiental y de seguridad, que también se extienden a las empresas tercerizadas.

52 La formación del sindicato de la segunda empresa tuvo otro desarrollo, ligado también a las características de su casa matriz. En este caso, la empresa tenía escasa tradición de diálogo, siguiendo las pautas generales que marcan las relaciones laborales del país de origen, lo que dificultó la organización sindical. La formación del sindicato se hizo lentamente, de forma casi clandestina, pero una vez conformado, fue aceptado por la empresa. El peso de la tradición sindical local y el hecho de que un contingente amplio de trabajadores de la empresa proviniera de empresas papeleras de larga trayectoria gremial, permitieron modificar las condiciones iniciales que la empresa intentó plasmar en el campo de las relaciones laborales. Actualmente, según los referentes entrevistados, el sindicato cuenta con un $95 \%$ de afiliación.

El sindicato de esta empresa tiene una estructura lineal, en claro contraste con la estructura vertical de la mayoría de los sindicatos uruguayos. La cúpula dirigente es una comisión de delegados. Otra característica de este sindicato es que su funcionamiento no se encuadra en las corrientes políticas características del movimiento sindical uruguayo. Un rasgo que comparten ambos sindicatos y que los diferencian del tradicional sindicato uruguayo, es que en ambas empresas resulta imposible, por razones tecnológicas, detener el funcionamiento de la planta. No se pueden realizar paros parciales ni totales por la complejidad y peligrosidad del proceso de trabajo. Esto implica que una medida de lucha tradicional del sindicalismo en general, el paro de actividades, sea - a entender de los referentes- muy difícil de implementar. Una solución parcial que encontraron estos sindicatos, fue la de trabajar con una dotación mínima y realizar asambleas fuera de la planta, pero, de todos modos, la efectiva implementación de las clásicas medidas de lucha del movimiento sindical se ve muy limitada.

Estas dificultades establecen diferencias importantes con el accionar del resto del sindicalismo uruguayo, generando polémicas y discusiones con sectores sindicales cercanos o afines. Pese a las mismas, ambos sindicatos participan activamente del plenario intersindical y en la negociación a nivel de rama, en la cual los altos salarios que se pagan en estas empresas inciden positivamente hacia la suba de salarios en otras empresas de la rama, manteniéndose siempre fuertes diferencias entre ambas realidades. La orientación estratégica de estos sindicatos prioriza la discusión y negociación antes que la utilización de medidas gremiales. Esta estrategia es posible en el marco de relaciones laborales en las cuales la empresa mantiene una actitud receptiva hacia las demandas sindicales, priorizando el diálogo y la negociación antes que la utilización de medidas represivas.

\section{Reflexiones finales}

55 Las organizaciones de alta confiabilidad se estructuran en torno al logro de altos niveles de confianza tanto externos como internos. Este primer nivel de análisis muestra los mecanismos que implementan estas empresas para alcanzar mejores niveles de confianza interna, a través de la implementación de políticas de formación, controles rigurosos y sistemas de recompensas y de reconocimientos que incentivan actitudes positivas en materia de seguridad. 

empresas uruguayas y a las empresas de la rama forestal instaladas anteriormente. En trabajos anteriores, mostramos como la mayoría de las empresas forestales de capital internacional instaladas en Uruguay, desarrollaron políticas de seguridad inspiradas en el modelo HRO, pero limitadas por el contexto productivo y laboral en el cual se implementaron. En este sentido, señalamos en trabajos anteriores que la estacionalidad de la producción y la precariedad de las formas de contratación de la fuerza de trabajo de la producción forestal, limitaron el alcance de estas políticas, que presuponen procesos de aprendizaje organizacional de largo aliento, altos niveles de participación de los trabajadores y un marco laboral estable. Las dos empresas analizadas parecen superar ampliamente las limitaciones encontradas en las empresas forestales, en la medida que resuelven muchas de las contradicciones señaladas. La formalización del empleo, los altos niveles de automatización de los procesos de trabajo y el marco de relaciones laborales donde predomina el diálogo y la negociación establece un contexto laboral y productivo mejor articulado con las modernas políticas de seguridad inspiradas en los modelos HRO. general, superar la lógica de "enclave" en la cual se han desarrollado estos dos emprendimientos. Los niveles de inversión, la tecnología desarrollada, la fuerte presencia en su origen de personal técnico y laboral de los países de origen, y el aislamiento geográfico en el cual desarrollan sus actividades, los convierten en un enclave más cercano a la realidad de los países desarrollados que a la latinoamericana. industria nacional se ve fuertemente limitada por las características económicas y tecnológicas en las cuales despliegan su actividad la mayoría de las empresas de este país. De todas maneras, la presencia de estas experiencias de tipo HRO impacta favorablemente en algunos sentidos. En primer lugar, empuja a un ajuste y actualización de todos los mecanismos de regulación del riesgo establecidos y a su efectiva aplicación. En segundo lugar, se muestra como ejemplo a ser tomado en cuenta en las negociaciones laborales en diferentes planos: salarial, institucional y de seguridad laboral. Por último, aporta experiencias e insumos técnicos valiosos para los especialistas y gestores en seguridad, en la medida en que encuentran un referente empírico para los diseños y aplicaciones de políticas de seguridad.

Esta lógica de enclave impacta también en las relaciones laborales, las estrategias sindicales y las formas de negociación colectiva. El modelo de relaciones laborales, en los casos estudiados, tiene más puntos de contacto con experiencias de países de altos niveles de industrialización, en los cuales el sindicalismo desarrolla una alta capacidad de negociación a partir de un perfil netamente corporativo y apolítico, en claro contraste con las tradiciones históricas del movimiento sindical. Estas orientaciones se enmarcan en tendencias que están presentes en otros sectores del movimiento sindical, pero que se expresan nítidamente en el caso de las empresas pasteras. A partir del año 2005, emergen en el sindicalismo uruguayo liderazgos sindicales menos articulados con las organizaciones políticas de izquierda, modalidad histórica predominante desde los años 60 en las relaciones sindicatos- partidos políticos. En el año 2005, cuando la izquierda llega al gobierno, muchos sindicatos recién formados y/o reconstituidos luego del debilitamiento del movimiento sindical producido por las políticas neoliberales entre 1990 y 2005, en sectores de escasa tradición sindical o en los nuevos 
sectores productivos que ocupan trabajadores jóvenes y de mayores niveles de calificación, desarrollaron líneas de acción con perfiles más corporativos y con un discurso menos apegado a la clásica lógica de la lucha de clases. Estas tendencias, si bien no son predominantes, tienen clara incidencia en las discusiones internas del movimiento sindical y en sus lineamientos estratégicos, lo que introduce cambios sustantivos en las formas de acción históricas del movimiento obrero uruguayo.

\section{BIBLIOGRAFÍA}

Avelardo, R. (2009). La expansión forestal en el Cono Sur: políticas públicas, intereses transnacionales y transformaciones territoriales. Revista Nueva Sociedad, 223, 76-93.

Agenda Forestal (2011). Agenda Florestal 2011 - Uruguay. Retirado de http:// www.uruguayforestal.com/informes/agenda-forestal-2011.pdf

Beck, U. (1998). La sociedad del riesgo. España: Editorial PAIDOS.

Boissières, I. (2007). Robustez organizacional y gestión del riesgo. In: La gestión del riesgo y las crisis. Personas, culturas organizacionales e instituciones. Buenos Aires: Editorial El Ateneo.

Bourrier, M. (2001). La fiabilité est une question d'organisation. In M. Bourrier (Dir.), Organiser la fiabilité (pp 9-38). Paris: L'Harmattan.

Cantero, J., \& Seijo, G. (2012). Rasgos ontológicos de las Organizaciones de Alta Confiabilidad (HROs): precisiones epistemológicas para la comprensión de un objeto de estudio en debate. Revista del Centro de Estudios de Sociología del Trabajo, 4, 69-96.

Coriat, B., \& Guennif, S. (2000). Incertitude, confiance et institution. In R. Laufer, \& M. Orillard (Dirs.), La confiance en question (pp. 215-244). Paris: L'Harmattan

De la Garza Toledo, E. (2010). Hacia un concepto ampliado de trabajo. Del concepto clásico al no clásico. México: Editorial Anthropos.

Douglas, M. (1985). Risk acceptability according to the social sciences. New York: Russel Sage Foundation.

Dourlens C., Galland J., \& Vidal-Naquet P. (1991). Introduction. In C. Dourlens, J. Galland, J. Theys, \& P. Vidal-Naquet (Eds.), Conquête de la sécurité, gestion des risques (pp. 11-42). Paris: L'Harmattan.

Fahlbruch, B., \& Wilpert, B. (1999). Sistem Safety, an Emerging Field for I/O Psychology. In C. Cooper, \& R. Robertson (Eds.), International Review of Industrial and Organizational Psychology (pp. 55-91). New York: Wiley.

Florit, P. (2013). La cadena forestal celulósica, contexto para el puerto de La Paloma. In D. Piñeiro (Coord.), Repercusiones de las inversiones forestales: la ampliación del puerto de la Paloma (pp. 13-42). Montevideo: Editorial CSIC-UdelaR.

Florit P., \& Piedracueva, M. (2016). Agronegocio y corporaciones transnacionales modelando el Uruguay dependiente. NóMadas. Critical Journal of Social And Juridical Sciences, 50(1), 299-326. doi: 10.5209/NOMA.54345 
Juncal, A., \& Fernandez, E. (2013). Valoración sobre el puerto multimodal de La Paloma. In D. Piñeiro (Coord.), Repercusiones de las inversiones forestales: la ampliación del puerto de la Paloma (pp. 39-86). Montevideo: Editorial CSIC-UdelaR.

La Porte, T. (2001). Fiabilité et légitimité soutenable. In M. Bourrier (Dir.), Organiser la fiabilité (pp. 71-107). Paris: L'Harmattan.

Luhmann, N. (1992). Sociología del riesgo. México: Universidad Iberoamericana.

Morales, V (2016). La dinámica del sector forestal en Uruguay. Revista Integración \& Comercio, 39, 192-195.

Paolino C., Pittaluga L., \& Mondelli M. (2014). Cambios en la dinámica agropecuaria y agroindustrial del Uruguay y las políticas públicas. Estudios y Perspectivas. CEPAL. Retirado de https:// es.scribd.com/document/236456462/Cambios-en-La-Dinamica-Agro-URUGUAY

Perrow, C. (1984). Normal Accidents. Living with High-Risk Technologies. New York: NJ, Basic Books.

Pou y Asociados (2016). Uruguay Forestal: principales tendencias del año 2016. Retirado de http:// www.uruguayforestal.com/informes/uruguayforestal16.pdf

Rochlin, G. (1996). New directions in Reliable Organizations Research and Future Directions. Journal of Contingencies and Crisis Management, 4(2), 55-60.

Tommasino H., Cortelezzi A., Ackermann M., Gorga L., Petraglia C., Souto G., Annuziatto W., \& Martin D. (2016). ¿Cómo impactó el crecimiento de la cadena forestal al agro y la economía uruguaya. Montevideo: MGAP.

Uruguay XXI (2016). Informe Mensual de Comercio Exterior. Retirado de https:// www.aduanas.gub.uy/innovaportal/file/16016/1/informe-mensual-de-comercio-exteriorjulio-2016.pdf

\section{RESÚMENES}

El trabajo se propone realizar un estudio de dos plantas de producción de pasta de celulosa que han tenido alto impacto en la realidad industrial uruguaya. Ambas plantas de origen internacional, con diferencias importantes en el funcionamiento de sus casas matrices, son consideradas como ejemplos de modelos HRO en la producción industrial nacional. El tipo de tecnología automatizada implementada ha reducido significativamente los riesgos clásicos de este trabajo. La hipótesis que se sostiene en este trabajo es que estos modelos requieren para su implementación un modelo de relaciones laborales diferente al que predomina en la mayoría de las empresas nacionales. Las altas condiciones de seguridad que se desarrollan en el sector están basadas en una estrategia de diálogo permanente por parte de la gerencia con un sindicato con una fuerte orientación corporativa, que prioriza las metas económicas y que tiende a reducir el componente ideológico en sus formas de acción.

O trabalho pretende realizar um estudo sobre duas fábricas de celulose que tiveram alto impacto na realidade industrial uruguaia. Ambas plantas, de origem internacional, com diferenças importantes na operação de suas casas matrizes, são consideradas exemplos de modelos HRO na produção industrial nacional. o tipo de tecnologia automatizada implementada reduziu significativamente os riscos clássicos deste trabalho. A hipótese que surge neste artigo é que esses modelos requerem um modelo de relações de trabalho para sua implementação que difere do prevalecente na maioria das empresas nacionais. As condições de segurança elevadas que são desenvolvidas no setor são baseadas em uma estratégia de diálogo permanente por parte da 
empresa com um sindicato com uma forte orientação corporativa, que prioriza os objetivos econômicos e tende a reduzir o componente ideológico em suas formas de ação.

Le travail présenté réalise une étude de deux usines de production de pâte de cellulose qui ont un impact important dans la réalité industrielle uruguayenne. Les deux usines d'origine internationale, avec d'importantes différences dans le fonctionnement de leurs sociétés mères, sont considérées comme des exemples de modèles HRO dans la production industrielle nationale. Le type de technologie automatisée mise en œuvre a considérablement réduit les risques classiques de ce travail. L'hypothèse du présent document est que ces modèles ont besoin, pour leur mise en œuvre, d'un modèle de relations de travail différent de celui qui prévaut dans la plupart des entreprises nationales. Les hautes conditions de sécurité développées dans le secteur reposent sur une stratégie de dialogue permanent de la part de la direction avec un syndicat fortement corporatif, qui priorise les objectifs économiques et tend à réduire la composante idéologique de ses formes de action.

The work intends to make a study of two cellulose pulp production plants that have had a high impact on the Uruguayan industrial reality. Both plants of international origin, with important differences in the operation of their parent companies, are considered as examples of HRO models in the national industrial production. The type of automated technology implemented has significantly reduced the classic risks of this work. The hypothesis that is sustained in this paper is that these models require for their implementation a model of labor relations different from that which prevails in most of the national companies. The high safety conditions that are developed in the sector are based on a strategy of permanent dialogue on the part of management with a union with a strong corporate orientation, which prioritizes economic goals and tends to reduce the ideological component in its forms of action.

\section{ÍNDICE}

Mots-clés: gestion, risque, syndicalisme, industrie, cellulose

Palabras claves: gestion, riesgo, sindicalismo, industria, celulosa

Palavras-chave: gestão, risco, unionismo, indústria, celulose

Keywords: risk, management, unionism, cellulose, industry

\section{AUTORES}

\section{FRANCISCO PUCCI}

Departamento de Sociología de la Facultad de Ciencias Sociales, UDELAR; Constituyente 1502, piso 5. CP 11.200 Montevideo, Uruguay

francisco.pucci@cienciassociales.edu.uy

\section{SOLEDAD NIÓN}

Departamento de Sociología de la Facultad de Ciencias Sociales, UDELAR; Constituyente 1502, piso 5. CP 11.200 Montevideo, Uruguay soledad.nion@cienciassociales.edu.uy 


\section{VALENTINA PEREYRA}

Departamento de Sociología de la Facultad de Ciencias Sociales, UDELAR; Constituyente 1502, piso 5. CP 11.200 Montevideo, Uruguay

valentinapereyra24@gmail.com 